Send your letters to the Editor, British

Dental Journal, 64 Wimpole Street, London

W1G8YS E-mail bdj@bda.org

Priority will be given to letters less than 500

words long. Authors must sign the letter,

which may be edited for reasons of space.

\section{A wider spectrum}

Sir, we are responding to the recent articles TMD and occlusion... by F. Luther (BDJ 2007; 202: E2, E3). While these papers draw on the multi-factorial aetiology of TMD, we know there is overwhelming evidence to suggest that TMD and related chronic oro-facial pain conditions like atypical facial pain are part of a wider spectrum of disorders with common psychological factors. ${ }^{1}$ Case-control studies ${ }^{2,3}$ that have examined the role of mechanical and psychological factors associated with TMD found that although cases had significantly increased pain on temporomandibular joint palpation, they also reported pain on palpation of placebo sites twice as frequently.

In addition, although cases had restricted mouth opening and deviation on jaw opening, there was no deterioration in corresponding function associated with these symptoms. Importantly, there were no differences between cases and controls in joint sounds, occlusion, facial trauma, missing teeth, prior dentistry and presence of dentures. The second, more recent case-control study $^{3}$ found that although mechanical factors like facial trauma and teeth grinding were associated with cases of TMD, equally important was the role of psychological factors like distress, sleep disturbance and aspects of illness behaviour.

Other studies ${ }^{4}$ have shown stronger relationships with psychological factors and while there was an association with mechanical factors, those such as trauma and third molar removal are not amenable to invasive intervention. Rather, primary care practitioners should recognise this condition early by taking a thorough history which will indicate the presence of other chronic pain disorders and psychological distress.

Patients should be given early intervention in the form of patient focused leaflets and counselling. More severely disabled patients need referral to a tertiary clinic for cognitive behaviour therapy for which there is good quality evidence from randomised controlled trials. ${ }^{5,6}$ Just as there is no justification for occlusal adjustments, ${ }^{7}$ there is also insufficient evidence for occlusal splints which do not address the problem in a holistic manner, which is essential. ${ }^{8}$

\section{R. Aggarwal}

\section{J. Zakrzewska}

\section{London}

1. Aggarwal V R, McBeth J, Zakrzewska J M et al. The epidemiology of chronic syndromes that are frequently unexplained: do they have common associated factors? Int J Epidemio/ 2006; 35: 468-476.

2. Dworkin S F, Huggins K H, LeResche L et al. Epidemiology of signs and symptoms in temporomandibular disorders: clinical signs in cases and controls. J Am Dent Assoc 1990; 120: 273-281.

3. Macfarlane TV, Gray R J M, Kincey J et al. Factors associated with the temporomandibular disorder, pain dysfunction syndrome (PDS): Manchester case-control study. Oral Dis 2001; 7: 321-330.

4. Huang G J, LeResche L, Critchlow C W et al. Risk factors for diagnostic subgroups of painful temporomandibular disorders (TMD). J Dent Res 2002; 81: 284-288.

5. Turner J A, Holtzman S, Mancl L. Mediators, moderators, and predictors of therapeutic change in cognitive-behavioral therapy for chronic pain. Pain 2007; 127: 276-286.

6. Gatchell R J, Stowell A W, Wildenstein L et al. Efficacy of an early intervention for patients with acute temporomandibular disorder-related pain: a one-year outcome study. J Am Dent Assoc 2006; 137: 339-347.

7. Koh H, Robinson P G. Occlusal adjustment for treating and preventing temporomandibular joint disorders. Cochrane Database Syst Rev 2003; 1: CD003812.

8. Forssell H, Kalso E. Application of principles of evidence-based medicine to occlusal treatment for temporomandibular disorders: are there lessons to be learned? J Orofac Pain 2004; 18: 9-22.

DOI: 10.1038/bdj.2007.235

\section{Fundamentally flawed}

Sir, the paper The use of acupuncture in controlling the gag reflex... (BDJ 2006; 201: 721-725) raises some interesting ideas but fundamentally misses some obvious points.

The gag reflex is not a stand alone reflex, it is dependent on whether the patient's swallowing reflex can adapt to the stressors to which it is subjected. If it can, there is no gagging, eg sword swallowing. If it cannot adapt, there is gagging.

In more severe cases interference with breathing reflexes initiated by obstructing the oral airway causes coughing and then gagging (we all know the importance of nose breathing when taking a full upper impression).

All the contributory factors to gagging the authors' list - and there are many others - affect swallowing. However, to try and distinguish between somatic and psychogenic gagging further blurs the simplicity of swallowing conditioning which leads to gagging problems. Pavlov's simple experiment would affect his dogs' swallowing!

Each patient has their own individual swallowing pattern which has variable adaption to a greater or lesser degree. Skilful less intrusive dentists encourage patients to have control over their swallowing.

I am surprised that there were no observations of the patient's swallowing movements. In my experience, ${ }^{1-3}$ all gaggers exhibit subconscious habitual clenched swallowing, which subsides on successful completion of treatment.

I note the authors state that in their technique, the needle is left in situ during impression taking, but in the results, they state that the impression was taken after acupuncture! If so, why was not the needle removed? Did any of the patients have impressions for a full upper denture? If so could they wear the full upper denture?

While a successful technique in the hands of dental acupuncturists, it is difficult to see how to set up a randomised controlled study taking into account all the variables.

\section{G. W. Wilks}

Billesdon

1. Wilks C G W. Classifications. Br Dent J 2005: 198: 561 .

2. Wilks C G W. Treatment of a dental phobic with pronounced aversion to rubber gloves by swallowing relaxation in two appointments. Br Dent J 1993; 175: 88-89.

3. Wilks C G W, Marks I M. Reducing hypersensitive gagging. Br Dent J 1983; 155: 263-265.

The author of the paper, Consultant Acupuncturist Palle Rosted, responds: Thank you for the comments on my paper. We agree that gagging is a complex problem, where the patient's reaction pattern and 
the dentist's approach to the problem are of importance. In the majority of cases, the dentist and the patient together manage to solve the problem. However, there remains a small group of patients who despite all efforts are unable to solve the problem. It is this group of patients this study concerns.

In this study, all patients had unsuccessfully received the dentist's standard treatment to control the gagging reflex, and the acupuncture was a supplement to this. Dr Wilks mentions a number of important factors for controlling the gag reflex, but as this treatment is a supplementary treatment, one must assume that most of these factors have been tried by the dentist in the past.

Regarding the description of the technique 'the needle is left in situ during impression taking and the impression was taken after acupuncture', the correct wording should have been: 'after stimulation the needle was left in situ during the impression and removed after the impression was taken'.

We have investigated the problem for various procedures, eg for a full upper denture and a bite-wing $x$-ray. However, to make the test group as homogeneous as possible, we decided only to include this group of patients.

We agree it would be difficult to carry out a proper randomised controlled study in this case; however, it needs to be done to establish, ultimately, if acupuncture is useful to treat this condition.

DOI: 10.1038/bdj.2007.236

\section{Undue prominence}

Sir, I have felt very uneasy about the uncritical publication of reports in the News section of the $B D J$ of studies published elsewhere. Most notably, the journal has published a string of reports in recent issues about possible links between periodontal diseases and other systemic conditions. In the absence of any critical attempt to appraise the clinical and scientific importance of these papers the journal risks giving undue prominence to research which while interesting and important is not in any way groundbreaking. I think it is important that more careful editorial consideration about the scientific and clinical importance of these news articles should be assessed. However, the papers suggesting no causal link between periodontal disease and other diseases, which are regularly featured in the 'Abstracts in other journals' section selected by $\mathrm{Dr}$ T. Watts, seem to inadvertently act as a nice balance to the News section.

In keeping with my general concern, I am sure that very many of your readers would have been extremely surprised, as was I, that a report that the gingival tissues produce cytokines which can cause bone resorption could be considered as 'News' suitable for a three column article. This particular information has been known for perhaps 35 years or more, as I am sure generations of dentists and hygienists will attest to having learned during their training.

I have no doubt that Dr Palmqvist's work (BDJ 2007; 202: 119) may likely have made a novel contribution to the over 2,000 existing papers in this field related to cytokines and the periodontal tissues, but what this is, is not stated in the article that you published. The weblink provided is for the Swedish Research Council, and I was only able to find details of projects in Swedish, which was not very helpful for me!

I appreciate that the choice of News articles is a difficult editorial issue, but please can we ask for more careful scientific appraisal when selecting articles for the News section, and avoid the uncritical publication of articles?

F. Hughes

London

The Editor-in-Chief responds: I thank Professor Hughes for his comments on the research items which we provide for readers' information and interest in the 'news' section. Reportage is by its nature 'uncritical', which is why the items are in 'news' and not in 'research'. However, the majority of items used in this way are derived from scientific journals with peer reviewing systems so that some element of judgement has been made on their worth before the content ever nears the BDJ.

Additionally one has to remember that the $\mathrm{BDJ}$ is read by the whole range of the profession from dental students to eminent academics, from general dental practitioners through to specialists and from DCPs to consultants; not everyone is at the same stage of learning and knowledge and we need to include content that will appeal across such diverse requirements. As Professor Hughes points out, in the same issue, 'balance' is provided by information elsewhere in the journal in a different style again in the form of abstracts.

DOI: 10.1038/bdj.2007.237

\section{Correction}

Please note that the letter Value of electives (BDJ 2007; 202: 114-115) inadvertently gave P. Carrotte as the sole author. The letter was in fact written by both Peter Carrotte and Dr Andrew Crothers, Director and Deputy Director of Elective Studies respectively. 